\title{
BMJ Open Use of autologous fat grafting for reconstruction postmastectomy and breast conserving surgery: a systematic review protocol
}

\author{
Riaz A Agha, ${ }^{1}$ Tim Goodacre, ${ }^{2}$ Dennis $\mathrm{P}$ Orgill ${ }^{3}$
}

To cite: Agha RA, Goodacre T, Orgill DP. Use of autologous fat grafting for reconstruction postmastectomy and breast conserving surgery: a systematic review protocol. BMJ Open 2013;3:e003709. doi:10.1136/bmjopen-2013003709

- Prepublication history and additional material for this paper is available online. To view these files please visit the journal online (http://dx.doi.org/10.1136/ bmjopen-2013-003709).

Received 3 August 2013 Revised 6 September 2013 Accepted 13 September 2013

${ }^{1}$ Department of Plastic Surgery, Stoke Mandeville Hospital, Aylesbury, Bucks, UK

${ }^{2}$ Department of Plastic Surgery, John Radcliffe Hospital, Oxford University Hospitals NHS Trust, Oxford, UK

${ }^{3}$ Division of Plastic Surgery, Brigham and Women's Hospital, Boston,

Massachusetts, USA

Correspondence to Dr Riaz A Agha; mail@riazagha.com

\section{ABSTRACT}

Introduction: There is growing interest in the potential use of autologous fat grafting (AFG) for the purposes of breast reconstruction. However, concerns have been raised regarding the technique's clinical effectiveness, safety and interference with screening mammography. The objective of this systematic review was to determine the oncological, clinical, aesthetic and functional, patient reported, process and radiological outcomes for AFG.

Methods and analysis: All original studies, including randomised controlled trials, cohorts studies, case-control studies, case series and case reports involving women undergoing breast reconstruction. All AFG techniques performed for the purposes of reconstruction in the postmastectomy or breast conserving surgery setting will be considered. Outcomes are defined within this protocol along; oncological, clinical, aesthetic and functional, patient reported, process and radiological domains. The search strategy has been devised to find papers about 'fat grafting and breast reconstruction' and is outlined within the body of this protocol. The full search strategy is outlined within the body of the protocol. The following electronic databases will be searched from 1 January 1986 to 6 June 2013: PubMed, MEDLINE, EMBASE, SCOPUS, CINAHL, PsycINFO, SciELO, The Cochrane Library, including the Cochrane Database of Systematic Reviews (CDSR), Cochrane Central Register of Controlled Trials (CENTRAL), Database of Abstracts of Reviews of Effect (DARE), the Cochrane Methodology Register, Health Technology Assessment Database, the NHS Economic Evaluation Databases and Cochrane Groups, ClinicalTrials.gov, Current Controlled Trials Database, the World Health Organisation (WHO) International Clinical Trials Registry Platform, UpToDate.com, NHS Evidence and the York Centre for Reviews and Dissemination. Grey literature searches will also be conducted as detailed in our review protocol. Eligibility assessment occurred in two stages, title and abstract screening and then full text assessment. Data were extracted and stored in a database with standardised extraction fields to facilitate easy and consistent data entry.

Ethics and dissemination: This systematic review will be published in a peer-reviewed journal. It will also be presented at national and international conferences in the fields of plastic, reconstructive and aesthetic surgery and at more general surgical and methodological conferences. It will be disseminated electronically and in print. Brief reports of the review findings will be disseminated directly to the appropriate audiences of surgeons and societies through email and other modes of communication. Updates of the review will be conducted to inform and guide healthcare practice and policy.

Protocol Registration: PROSPERO-National Institute of Health Research (NIHR) Prospective Register of Systematic Reviews (CRD42013005254)

\section{BACKGROUND \\ Breast cancer and reconstruction}

Breast cancer is the most common cancer in the UK and is by far the most common cancer among women, where it accounts for $31 \%$ of all new cancer cases with a life-time incidence of 1 in $8 .^{1}$ Approximately 50000 women are diagnosed with breast cancer each year, 16000 of whom undergo mastectomy and there are approximately 12000 deaths from the disease. ${ }^{2}$ In the USA, the National Cancer Institute predicts 232340 new cases and 39620 deaths from breast cancer in 2013 with over 96000 undergoing breast reconstruction following surgery. Surgical treatment options include mastectomy or breast conserving surgery (BCS), which is either quadrantectomy or lumpectomy plus axillary node dissection if nodal disease exists, often combined with radiotherapy (RT). Veronesi et $a l^{4}$ showed through a seminal randomised controlled trial beginning in 1973, comparing mastectomy with BCS, that long-term survival (after 20-year follow-up) was no different for those with tumours $<2 \mathrm{~cm}$ in diameter. Hence, BCS has become the treatment of choice for this group. $^{56}$ 
The mix of patients undergoing mastectomy or various forms of BCS leaves the reconstructive surgeon facing a variety of defects in the group of patients wanting reconstruction. There is a need for not just restoring volume but creating an aesthetically pleasing shape and also dealing with the aftermath of RT including pain, retraction, fibrosis, oedema, telangiectasia, atrophy as captured in the Late Effects Normal Tissue/Subjective Objective Management Analytic (LENT/SOMA) system for grading RT side effects. ${ }^{7}$ Reconstruction of the breast can occur either immediately postmastectomy or in a delayed setting several months or years later. Reconstructive options include implants or with autologous tissue as either a pedicled or free flap containing skin, fat and a variable amount of muscle harvested as a composite block of tissue. Existing reconstructive options are not without morbidity. Mioton $e t a l^{8}$ reviewed over 13000 breast reconstructions in the USA from 2006 to 2010 using the prospective and multicentre American College of Surgeons-National Surgical Quality Improvement Program (ACS-NSQIP) database. Autologous reconstructions (using mostly tissue from the abdomen or back) were performed in $25 \%$ of patients and implants were the reconstructive modality in $75 \%$. Autologous reconstruction patients had higher rates of overall complications ( $12.5 \%$ vs $5.4 \%)$, wound infection (5.5\% vs $3.5 \%)$, flap/prosthesis failure $(3.1 \%$ vs $0.9 \%)$ and reoperation (9.6\% vs $6.8 \%)$. There are of course general complications of major surgery and prolonged length of stay (3-5 days typically) such as deep vein thrombosis, pulmonary embolism, pneumonia and the need for blood transfusions. $^{9}$

Implant-based reconstructions include complications, such as implant exposure/extrusion, rupture, deformation/distortion, rippling, migration and discontent among patients with implant animation and edge visibility. The placement of the implant itself can lead to reduced or absent sensation at the nipple in one in seven women. ${ }^{10}$ Furthermore, the body's natural reaction to a foreign body is to form a fibrous capsule around it as part of the healing process. This fibrous capsule shrinks over time (capsular contracture), which can lead to implant prominence, distortion of aesthetics (especially symmetry), increased palpability and pain. ${ }^{10}$ In its core study presentation to the Federal Drug Administration (FDA), Allergan's 10-year cumulative risk study found that $24.6 \%$ of patients who underwent implant-based reconstruction developed capsular contracture necessitating implant removal and/or replacement. ${ }^{11}$ Implanting foreign bodies to fill space and provide volume and shape is felt to be unnatural by some patients and underscores their refusal to proceed with implant-based reconstruction. ${ }^{12}$ Such reservations were reinforced during the silicone implant scandal in the 1990s and has not been helped by the more recent poly implant prothese (PIP) implant scandal $^{13}$ and to a lesser extent the metal on metal hip replacement controversy. ${ }^{14}$

Another technique that has received much recent focus, is autologous fat grafting (AFG), where the patient's own fat is harvested using a liposuction technique, typically from the abdomen or thighs and then transplanted into the cavity left by removal of the gland postmastectomy or BCS. It holds the potential of reconstruction using nature's choice of filler-body fat, which is easily harvestable, often in good supply, where the donor site defect could be appreciated by patients with minimal scarring, no foreign body reaction and the potential for boosting skin trophicity and rejuvenation by combating the signs and symptoms of RT damage and aging, all performed as a day-case procedure. ${ }^{15} 16$

\section{The history of fat grafting}

In 1893, Neuber described the first reported case of autologous fat transfer for the purposes of reconstruction, implanting upper arm fat tissue to correct a depression in the face. ${ }^{17}$ In 1895 , Czerny performed the first breast reconstruction when he transplanted a large lipoma from the dorsal flank to the breast. ${ }^{18}$ In the 1950 s technical problems with harvesting and viability were brought into sharp focus when a study showed minimal graft survival 1 year post-transplantation. ${ }^{19}$ The corollary was that fat grafting fell out of favour at a time when synthetic implants were on the rise. The technique lay essentially dormant for a while but was revived in the early days of liposuction following Illouz's ${ }^{20}$ seminal paper describing the ability to remove fat cells from small port incisions using a cannula. Liposuction offered surgeons not just a method of body contouring but access to nature's own choice of filler-body fat through a low morbidity and safe approach that resulted in minimal scarring.

In 1987, Bircoll ${ }^{21}$ described the first reported case of harvesting fat using a liposuction technique with subsequent placement into the breasts using small syringes. This was performed in 1984 on a 20-year-old Caucasian woman who suffered a dog bite in the right thigh but who also had bilateral breast hypoplasia-with the harvested fat being used to treat both areas. ${ }^{21}$ Despite significant advances, obtaining consistently good aesthetic and reconstructive results for fat grafting to the breast remained challenging with complications such as cyst formation, fat necrosis and calcification relatively frequent compared with other body areas and volume maintenance being major hurdles. ${ }^{22}{ }^{23}$ This has been put down to the technique not being well understood at the time with the many technical factors influencing graft take and maintenance not being appreciated. ${ }^{25}$

\section{Controversy and prohibition of fat grafting}

In 1987, following concerns that potential scarring and calcifications could mask the detection of breast cancer and that adipocytes might directly stimulate the formation of cancer, the American Society of Plastic and Reconstructive Surgeons (ASPRS) Ad Hoc Committee on New Procedures prohibited the use of AFG to the female breast through a position statement which stated 
The committee is unanimous in deploring the use of autologous fat injection in breast augmentation. ${ }^{25}$

\section{The advent of structural fat grafting}

In the period that followed, Coleman ${ }^{26}$ developed the concept of structural fat grafting. The principle being that fat must be transplanted in small aliquots using multiple tunnels, in a multilayered and multidirectional way, formed by thin cannulas and syringes. This maximises the number of adipocytes in contact with the host tissue, optimising their chances of receiving sufficient nutrition and immobilisation to survive and become incorporated into the recipient site. ${ }^{27}$ Any fat that necroses, can cause an inflammatory reaction, with the sequelae of fibrosis and/or cystic formation plus or minus calcification and potential local infection. ${ }^{28}$ These principles are particularly important in the breast, where unlike the other anatomical areas, fat is being implanted into a loose and poorly vascularised space postmastectomy or BCS. ${ }^{26}$

A number of studies subsequently showed structural fat grafting to the breast to be efficacious. ${ }^{29-31}$ In 2009, the American Society of Plastic Surgeons (ASPS) Fat Grafting Task Force announced that fat grafting to the breast is not a strongly recommended procedure, as there is limited scientific data on safety and efficacy. ${ }^{23}$ They also stated that: "there appears to be no interference with breast cancer detection."

\section{Growing knowledge on fat grafting}

There has been much focus on how to boost fat graft survival including the development of centrifugation techniques for distilling the fat fraction from the harvested fluid (which will also contain blood residues in the precipitate and an oily supernatant composed of lysed cells and free triglycerides) and the understanding that adipose tissue is an endocrine organ with distinct circadian mechanisms and is rich in mesenchymal stem cells (MSCs). ${ }^{16} \quad 32$ Such adipose-derived stem cells (ADSCs) can differentiate into a range of cells types (eg, chondrocytes, myocytes and osteoblasts) and secrete angiogenic factors such as vascular endothelial growth factor. $^{32} 33$

ADSCs have now become the most studied adult stem cell and are thought to play a key role in the restorative, reconstructive and sustainable qualities of transferred fat. $^{1634-38}$

\section{Current controversy and concerns}

Pearl $e t a l^{39}$ have cited concerns with respect to fat grafting into an area of previous malignant change. They highlighted basic science and animal studies which found that engrafted MSCs were less able to regulate growth patterns and how this could potentially lead to breast cancer recurrence. ${ }^{37}{ }^{40}$ Wang $e t a l^{41}$ have emphasised the role that adipose tissue may play in breast cancer progression and metastasis. Recent studies have shown that adipokine signalling may foster cancer initiation and progression with adipose tissue progenitors cooperating in breast cancer angiogenesis, growth and metastatic progression. ${ }^{42}$ There are also concerns about how AFG may lead to mammographic confusion with $16.7 \%$ of patients in one sample showing the subsequent development of clustered microcalcifications. ${ }^{43}$ The greatest concern thus comes in the use of AFG-which stimulates angiogenesis-to reconstruct what was a tumour bed that is, postmastectomy or BCS.

\section{What have we learnt from prior systematic reviews?}

The table below summarises previous systematic reviews assessing AFG to the breast.

The quality of the previous systematic reviews is assessed in table 1 below using the validated assessment

Table 1 Quality of prior systematic review according to AMSTAR

\section{AMSTAR criterion}

(1) Was an 'a priori' design provided?

(2) Was there duplicate study selection and data extraction?

(3) Was a comprehensive literature search performed?

(4) Was the status of publication (ie, grey literature) used as an inclusion criterion?

(5) Was a list of studies (included and excluded) provided?

(6) Were the characteristics of the included studies provided?

(7) Was the scientific quality of the included studies assessed and documented?

(8) Was the scientific quality of the included studies used appropriately in formulating conclusions?

(9) Were the methods used to combine the findings of studies appropriate?

(10) Was the likelihood of publication bias assessed?

(11) Was the conflict of interest stated?

Total

\begin{tabular}{|c|c|c|c|}
\hline Gutowski $^{23}$ & Rosing et $a l^{49}$ & Claro et $a^{48}$ & Leopardi et $a^{47}$ \\
\hline$\checkmark$ & $\checkmark$ & $\checkmark$ & $\checkmark$ \\
\hline Unknown & $x$ & $\checkmark$ & $x$ \\
\hline$x$ & $x$ & $\checkmark$ & $\checkmark$ \\
\hline$x$ & $x$ & $\checkmark$ & $\checkmark$ \\
\hline$x$ & $x$ & $x$ & $x$ \\
\hline$x$ & $\checkmark$ & $\checkmark$ & $\checkmark$ \\
\hline$x$ & $x$ & $\checkmark$ & $\checkmark$ \\
\hline$\checkmark$ & $x$ & $\checkmark$ & $\checkmark$ \\
\hline $\mathrm{N} / \mathrm{A}$ & $\mathrm{N} / \mathrm{A}$ & $x$ & $x$ \\
\hline$x$ & $x$ & $x$ & $x$ \\
\hline$\checkmark$ & $\checkmark$ & $x$ & $x$ \\
\hline $3 / 10^{*}$ & $3 / 10^{*}$ & $7 / 11$ & $6 / 11$ \\
\hline
\end{tabular}

*Scored out of 10 since criterion 9 will not apply to these studies.

AMSTAR, Assessment of Multiple Systematic Reviews; N/A, not applicable. 
tool 'Assessment of Multiple Systematic Reviews (AMSTAR), ${ }^{44-46}$

From table 2, it can be noted that two reviews do not state the years searched or only searched a narrow range of databases. ${ }^{23} 49$ All the reviews were restricted to the English language, which creates substantial language bias. All of these reviews suffer from significant heterogeneity across the included studies with cosmetic as well as reconstructive indications among populations with congenital and acquired defects or use of cointerventions such as simultaneous implant or flap-based procedures along with AFG.

Many of the included studies did not report on patient satisfaction and those that did, did not use a standardised and validated instrument for such measurement. The follow-up periods were also highly variable, so the time point at which outcomes are measured is highly variable.

\section{Why is it important to do this systematic review?}

AFG is an active research front. A basic search using the database SCOPUS for 'fat grafting' reveals how research and interest in this area has increased in recent years (figure 1).

Since the most recent systematic review looking at the use of AFG in breast reconstruction by Claro $e t ~ a l^{48}$ concluded its search in June 2011, there have been approximately 235 more articles published in this area. A new systematic review is needed to update our understanding of this rapidly evolving reconstructive technique and potentially answer the questions previous studies have.

\section{OBJECTIVES}

Our objective is to perform a comprehensive systematic review of AFG for the purposes of breast reconstruction with a particular focus on safety, efficacy and radiological outcomes.

\section{Primary objectives}

In the context of using AFG for breast reconstruction in women postmastectomy or post-BCS, we aim to determine its

1. Oncological outcomes

2. Clinical outcomes

3. Aesthetic and functional outcomes

4. Patient-reported outcomes

5. Process outcomes

6. Radiological outcomes

\section{Secondary objectives}

1. To determine optimal methods of fat harvesting, preparation and injection.

2. To determine the indications for the procedure.

3. To help refine patient selection for the procedure.

\section{METHODS}

This review will be conducted in line with the recommendations specified in the Cochrane Handbook for Intervention Reviews V.5.1.0 and is AMSTAR compliant. ${ }^{50}$ It will be reported in line with the Preferred Reporting Items for Systematic Reviews and Meta-Analyses (PRISMA) statement. ${ }^{51}$ This protocol has been developed a priori and registered on the National Institute of Health Research (NIHR) Prospective Register of Systematic Reviews PROSPERO CRD42013005254. ${ }^{52}$

\section{Criteria for selecting studies for this review}

The following inclusion and exclusion criteria were explicitly formulated to minimise heterogeneity and address the research questions asked.

\section{Types of studies}

All original studies, including randomised controlled trials, cohorts studies, case-control studies, case series and case reports. Hence, levels of evidence (LoE) 1-5 (excluding expert opinion) as defined by the Oxford Centre for Evidence-Based Medicine. ${ }^{53}$ Only articles which mentioned one or more of the outcomes of interest will be included. Unpublished trials and reports would be included if the methodology and data are accessible in written form.

Duplicate studies will be excluded as will; costeffectiveness studies, those that did not contain an indication for the procedure, where original data is not reported such as review articles, editorials/discussions/ commentaries, letters containing only viewpoints or purely technical descriptions.

\section{Types of participants}

Participants were women undergoing immediate or delayed breast reconstruction following a mastectomy, a quadrantectomy, wide local excision or a lumpectomy for the treatment of breast cancer. Male and transgender cases will be excluded.

\section{Types of interventions}

Autologous fat transfer techniques performed for the purposes of reconstruction following oncological surgery would be considered. We will include total breast reconstruction postmastectomy and BCS using AFG (primary reconstruction) and secondary reconstruction following initial reconstruction with implants or flaps. Studies involving cadaveric grafts or pedicled fat flaps will be excluded. Studies where fat grafting is used as a cointervention or simultaneously in combination with another reconstructive technique (such as implants or flaps) or used as a salvage procedure for failed reconstructions or used purely for nipple reconstruction will be excluded. Studies where fat grafting was used for the purposes of cosmetic breast augmentation or to reconstruct traumatic breast defects (such as breast amputations, ballistic or blast trauma or burns) will be excluded. 
Table 2 Prior reviews of autologous fat grafting (AFG) to the breast

\begin{tabular}{|c|c|c|c|c|}
\hline Review & $\begin{array}{l}\text { Databases included and years } \\
\text { searched }\end{array}$ & Inclusion criteria & Studies included & Key findings \\
\hline $\begin{array}{l}\text { Leopardi } \\
\text { et } a l^{47}\end{array}$ & $\begin{array}{l}\text { PubMed, EMBASE, Current } \\
\text { Contents, The Cochrane Library } \\
\text { and the York Centre for Reviews } \\
\text { and Dissemination. } \\
\text { January } 2001 \text { to } 14 \text { January } \\
2009\end{array}$ & $\begin{array}{l}\text { Comparative studies comparing the safety and/ } \\
\text { or effectiveness of AFG with saline or cohesive } \\
\text { silicone implants (with either smooth or textured } \\
\text { walls) in adult women undergoing breast } \\
\text { augmentation for cosmetic indications (not } \\
\text { reconstructive) }\end{array}$ & $\begin{array}{l}532 \text { found in the } \\
\text { search and } 18 \text { were } \\
\text { included in the review }\end{array}$ & $\begin{array}{l}\text { Complications associated with AFG occurred in } \\
\text { only a small proportion of patients, including fat } \\
\text { necrosis, cysts and lumps. Reabsorption of fat } \\
\text { occurred to varying degrees. Patient satisfaction } \\
\text { following AFG was high with volume limitation } \\
\text { being the main discomfort. No data examining } \\
\text { the effect of complications such as } \\
\text { microcalcification on long-term mammographic } \\
\text { and cancer-related outcomes was identified }\end{array}$ \\
\hline $\begin{array}{l}\text { Claro } \\
\text { et } a l^{48}\end{array}$ & $\begin{array}{l}\text { MEDLINE, EMBASE, SciELO } \\
\text { and the Cochrane Library, July } \\
1986 \text { to June } 2011\end{array}$ & $\begin{array}{l}\text { Both augmentation and reconstructive } \\
\text { indications were included }\end{array}$ & $\begin{array}{l}302 \text { articles found in } \\
\text { the search and } 60 \\
\text { were included in the } \\
\text { review ( } 4601 \text { patients) }\end{array}$ & $\begin{array}{l}\text { The incidence of clinical complications was } \\
3.9 \% \text { of } 3015 \text { women ( } 21 \text { studies)-the majority } \\
\text { being induration and palpable nodularity. } \\
\text { Radiographic abnormalities were found in } 13 \% \\
\text { of } 2560 \text { patients ( } 17 \text { studies), the majority were } \\
\text { cysts. Local recurrence of breast cancer } \\
\text { occurred in } 2.3 \% \text { of } 616 \text { women (three studies). } \\
\text { The authors concluded that oncological safety } \\
\text { remains unclear }\end{array}$ \\
\hline $\begin{array}{l}\text { Rosing } \\
\text { et } a l^{49}\end{array}$ & $\begin{array}{l}\text { MEDLINE only } \\
\text { Time limits not stated }\end{array}$ & $\begin{array}{l}\text { Inclusion criteria not well defined. Excluded } \\
\text { studies that exclusively used AFG for contour } \\
\text { irregularities postbreast reconstruction }\end{array}$ & $\begin{array}{l}115 \text { articles found in } \\
\text { the search and } 17 \\
\text { included in the review } \\
\text { (1658 patients) }\end{array}$ & $\begin{array}{l}\text { The majority of patients included were satisfied } \\
\text { with the results but nine studies did not report } \\
\text { patient satisfaction }\end{array}$ \\
\hline Gutowski $^{23}$ & $\begin{array}{l}\text { PubMed and the Cochrane } \\
\text { Database of Systematic Reviews } \\
\text { Time limits not stated }\end{array}$ & $\begin{array}{l}\text { Search limits restricted results to } \\
\text { English-language articles that were indexed as } \\
\text { human studies, clinical trials, randomised } \\
\text { controlled trials, systematic reviews, case } \\
\text { series, or case reports. French language articles } \\
\text { were included if they were relevant to the } \\
\text { breast, which was the main focus of the task } \\
\text { force }\end{array}$ & $\begin{array}{l}187 \text { found in the } \\
\text { search and } 110 \\
\text { included in the review }\end{array}$ & $\begin{array}{l}\text { The authors recognise the lack of strong data } \\
\text { but recommended that fat grafting may be } \\
\text { considered for breast augmentation and } \\
\text { correction of defects associated with medical } \\
\text { conditions and previous breast surgeries; } \\
\text { however, results are dependent on technique } \\
\text { and surgeon expertise, which is not } \\
\text { standardised. Patient satisfaction was typically } \\
\text { good to excellent. Infection being a primary } \\
\text { concern, the need for sterile technique was } \\
\text { emphasised. Other complications included } \\
\text { seroma and haematoma. Two cases of breast } \\
\text { cancer were reported after fat grafting. The } \\
\text { review recommended that caution should be } \\
\text { exercised when considering high-risk patients } \\
\text { (ie, those with risk factors for breast cancer: } \\
\text { BRCA-1, BRCA-2 and/or personal or familial } \\
\text { history of breast cancer). } \\
\text { However there were no reports suggesting an }\end{array}$ \\
\hline
\end{tabular}




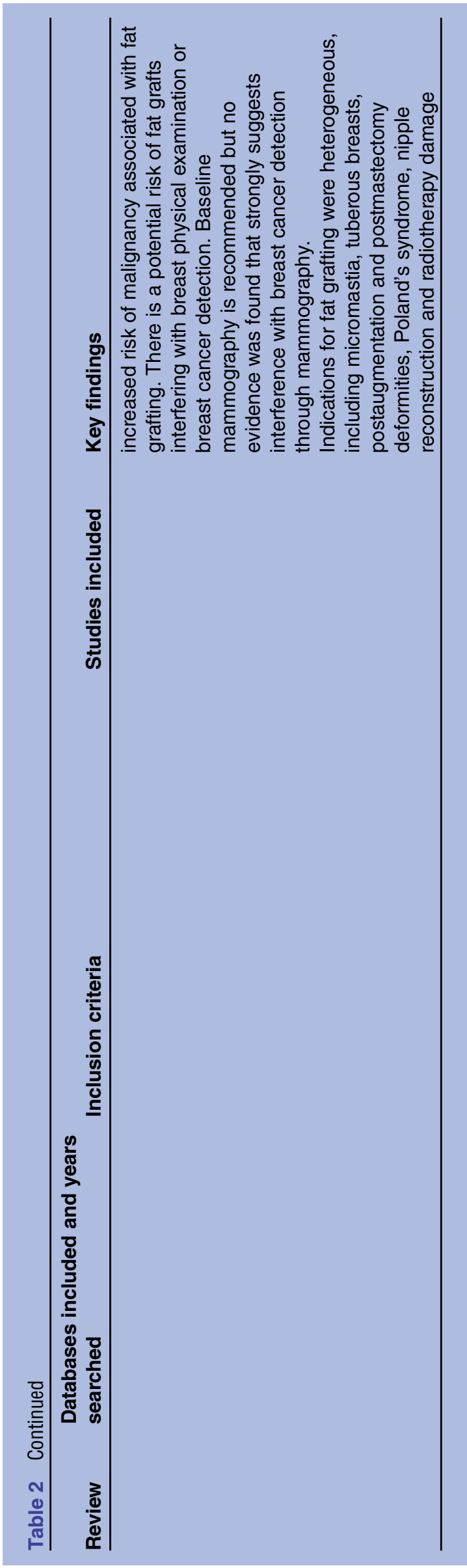

Types of comparators

Potential comparators include implant and flap-based reconstructions as well as no reconstruction at all.

\section{Types of outcome measures}

The outcomes of interest are defined along six distinct domains

1. Oncological outcomes: Defined as the incidence of new primary of recurrent breast cancer.

2. Clinical outcomes: Defined as the incidence of intraoperative and postoperative complications such as local infection, fat necrosis, oil cysts and palpable nodules. Complications will be graded using the validated Clavien-Dindo classification system, which assesses the therapeutic consequences of complications. ${ }^{54}$

3. Aesthetic and functional outcomes: Defined as clinician satisfaction with the results (subjective) as measured through questionnaire, visual analogue or other scale or Netscher score ${ }^{55}$ as well as more objective measures such as changes in LENT-SOMA score. ${ }^{7}$

4. Patient-reported outcomes: Defined as patient satisfaction with the procedure as measured by questionnaire, visual analogue or other scale or more formal instruments like BREAST-Q. ${ }^{56}$

5. Process outcomes: This refers to the number of sessions needed to achieve a satisfactory outcome, expressed as a mean together with a range for the group.

6. Radiological outcomes: Incidence of radiological abnormalities, such as calcific deposits, microcalcifications, cysts and other masses that may potentially interfere with mammographic screening.

\section{Search methods for identification of studies \\ Electronic searches}

Electronic databases will be searched from 1 January 1986 to 6 June 2013. The year 1986 was chosen as the start date by a multilingual information specialist with 8 years experience of searching Biomedical, Economic and Sociological databases, conducting approximately 80-100 literature searches per year: PubMed, MEDLINE, EMBASE, SCOPUS, CINAHL, PsycINFO, SciELO, The Cochrane Library including the Cochrane Database of Systematic Reviews (CDSR), Cochrane Central Register of Controlled Trials (CENTRAL), Database of Abstracts of Reviews of Effect (DARE), the Cochrane Methodology Register, Health Technology Assessment Database, the NHS Economic Evaluation Databases and Cochrane Groups, ClinicalTrials.gov, Current Controlled Trials Database, the World Health Organisation (WHO) International Clinical Trials Registry Platform, UpToDate. com, NHS Evidence and the York Centre for Reviews and Dissemination.

\section{Search terms and keywords}

The search strategy has been devised to find papers about 'fat grafting and breast reconstruction'. A search will be conducted using appropriate keywords in the English language combined with Boolean logical 
Figure 1 Number of articles published per year and indexed by SCOPUS under the search term "fat grafting".

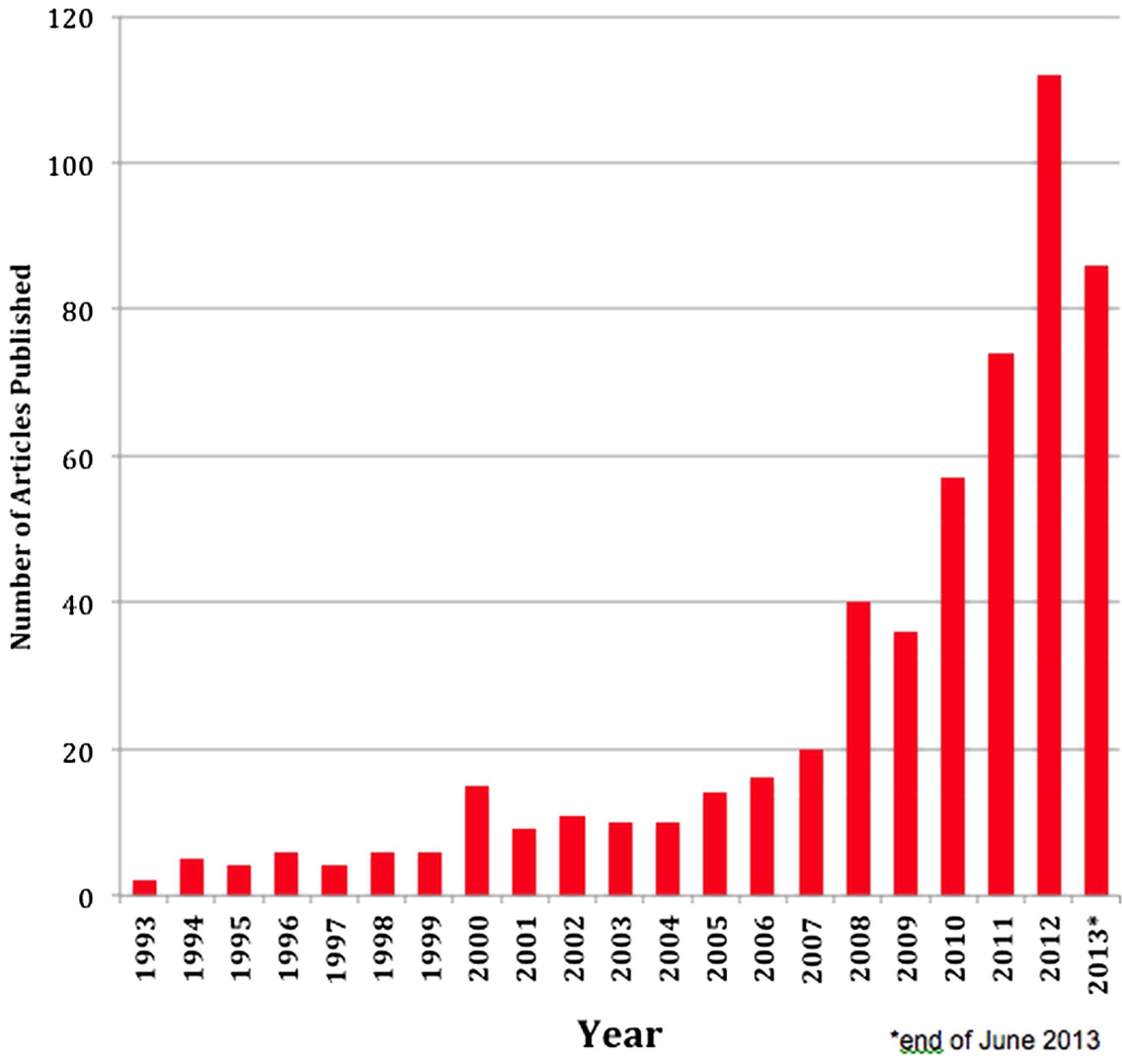

operators typically as follows: lipostructuring OR lipotransfer OR lipomodelling OR lipomodeling [Title/Abstract] OR "adipose tissue/transplantation" [MeSH Terms] OR fat OR "autologous fat" OR "adipose tissue" OR "body fat" OR "tissue adipose" OR "fatty tissue" [Title/Abstract] OR "adipose tissue" [MeSH Terms] AND (autograft* OR autotransplant* OR graft* OR transplant OR transplantat* OR injection OR transfer OR lipofilling [Title/Abstract]) AND (mammoplast* OR mammaplast* [Title/Abstract]) OR "mammaplasty" [MeSH Terms]) OR ("breast reconstruction" OR "breast reconstructed" OR "breast augmentation" OR "breast enlargement" OR "breast surgery" [Title/Abstract]), adapted to the appropriate syntax of each database. In all databases free text and the relevant database thesaurus terms will be used. The thesaurus terms will be exploded to capture narrower terms.

Reports will not be excluded based on their publication status. The search will not be limited by language. Where a non-English language article is found, it will proceed to title and abstract screening (since the abstract will be in English). If the full paper is required to determine its eligibility for final inclusion, the authors of the manuscript will be contacted where possible to provide an English language version of their article or language translation will occur through a native speaker. If these options are not possible, Google Translate (Google, Mountain View, California, USA) will be used. Google translate is based on statistical machine translation and according to Google, looks for patterns in hundreds of millions of documents that have already been translated by human translators to determine what an appropriate translation should be. ${ }^{57}$ It has recently been recognised as an approach to minimise language bias in systematic reviews. ${ }^{58}$

\section{Searching other resources}

Grey literature searches will include conference proceedings from the ACS and ASPS Annual Congresses in 2012 and the European Plastic Surgery Research Council (EPSRC) Annual Meetings of 2012 and 2013 (to capture recent as yet unpublished studies). Other literature to be searched will include the Plastic Surgery textbook Grabb and Smith's Plastic Surgery-6th edition, a core text in the specialty. ${ }^{59}$ We will attempt to contact researchers who are active in this field for information about further published or unpublished studies. A link to the PROSPERO record for the protocol will also be distributed through Twitter using the lead author's account and a call for unpublished work made. In addition, references for all included papers and prior systematic reviews will be searched for any relevant studies that were not already captured through our search.

\section{Identification and selection of studies}

Studies identified through the electronic and manual searches will be listed with citation, titles and abstracts from all databases being populated into a Microsoft Excel 2011 database and duplicates excluded (Microsoft, 
Redmond, Washington, USA). The eligibility process will be conducted in two distinct stages

1. Titles and abstracts will be screened by two researchers acting independently. A final list will be agreed with discrepancies resolved by consensus. If any doubt about inclusion exists, the article will proceed to the next stage.

2. The full-text version of the articles passing title and abstract screening will be downloaded and further assessed for eligibility by two researchers acting independently. Discrepancies will be resolved by consensus. If this is not possible, one of the senior authors will be asked to make a judgement on the article.

Multiple reports of the same study will be linked together. Where required, correspondence with the relevant investigators would take place to clarify study eligibility and results or if the article was not readily accessible. Once final decisions on study inclusion had been made, the authors will proceed to data extraction.

\section{Data extraction, collection and management}

Data extraction will be performed independently by two researchers and then discrepancies will be resolved by consensus. If this is not possible, one of the senior authors will be asked to make a judgement on the data entered.

Extraction of data will occur by developing a database using Microsoft Excel 2011 (Microsoft, Redmond, Washington, USA) with standardised extraction fields where data can be inputted from each study in turn. The following data will be extracted.

- Author names, countries and year of publication

- Study design and LoE according to Oxford Centre for Evidence-based Medicine

- Conflicts of interest and funding

- Number of participants

- Number of breasts treated

- Age of participants expressed as mean or median with a range if provided

- Previous oncology surgery-mastectomy, quadrantectomy, wide local excision or lumpectomy

- Prior adjuvant RT

- Previous breast reconstruction procedure(s)

- Time interval between oncology surgery and fat grafting

- Donor site(s) used

- Technique-recipient site preparation, graft harvest, preparation and injection

- Mean volume of fat injected per breast

- Mean follow-up length

- Loss to follow-up expressed as a percentage

- Oncological, clinical, aesthetic, functional, patient reported, process and radiological outcomes as defined above

\section{Assessment of study quality and bias in included studies}

The extent to which the results from this systematic review can confidently be applied in practice depends in part on the methodological rigour with which contributing studies were conducted. The methodological quality of studies will be assessed using the Grading of Recommendation Assessment, Development and Evaluation (GRADE) system. ${ }^{61}{ }^{62}$ GRADE offers four LoE quality: high, moderate, low and very low. Randomised trials begin as highquality evidence, observational studies as low-quality evidence and case series and case reports as very low-quality evidence.

Quality may be downgraded as a result of following five domains

- Limitations in study design or implementation (high risk of bias)

- Inconsistency in results

- Indirectness of evidence

- Imprecision of estimates (wide CIs)

- Publication bias

Quality may be upgraded because of three domains

- A very large magnitude of effect

- A dose-response gradient

- All plausible biases would reduce an apparent treatment effect

For randomised controlled trials, we will extract whether clinically relevant outcomes are reported and compare trial protocols with subsequent publications where available. Key missing information across all study types such as complication rates and follow-up times will be documented and assessed.

\section{Strategy for data synthesis and statistical analysis}

Outcomes of interest will be tabulated and shown in descriptive or numeric form as appropriate and summarised. Using Review Manager V.5.2.6 (RevMan), ${ }^{62}$ an assessment of heterogeneity in comparative studies will be made. $^{62}$ If high, meta-analysis will not be performed. Previous reviews have not conducted meta-analysis. ${ }^{47-49} 61$

\section{Subgroup analysis}

We will perform an additional analysis to separate total breast reconstructions using AFG postmastectomy, from its use to correct contour deformities postimplant, flapbased reconstructions or BCS alone.

\section{Dissemination}

This systematic review will evaluate the use of AFG for breast reconstruction. Hence it may influence the management of patients with breast cancer postmastectomy or BCS and the reconstructive options offered to them. Based on the results of this systematic review, independent analysis and recommendations will be made to clinicians, researchers, plastic surgical societies and policy makers. Authors of position statements and guidelines relating to AFG will be informed of the results directly. It will be published in the English language in a peerreviewed journal and the authors will endeavour to respond to any commentary generated. It will also be presented at national and international conferences in the fields of Plastic, Reconstructive and Aesthetic 
Surgery and at more general surgical and methodological conferences. It will be disseminated electronically and in print to leading researchers in the field. Brief reports of the review findings will be disseminated directly to the appropriate audiences and societies through email and other modes of communication. Updates of the review could be conducted to inform and guide healthcare practice and policy should the need arise.

Contributors RAA contributed to concept and methods, writing and approving protocol. TG was involved in steerage on methodology, writing input and approving protocol. DPO contributed to concept, steerage on methodology, writing input and approving protocol.

Funding This research received no specific grant from any funding agency in the public, commercial or not-for-profit sectors.

Competing interests None.

Ethics approval In line with guidance issued jointly by INVOLVE and the National Research Ethics Service (NRES), ethical approval is not needed for systematic reviews, even ones which involve patients and members of the public in a planning and advisory capacity. (INVOLVE. Patient and public involvement in research and research ethics committee review, 2009)

Provenance and peer review Not commissioned; externally peer reviewed.

Open Access This is an Open Access article distributed in accordance with the Creative Commons Attribution Non Commercial (CC BY-NC 3.0) license, which permits others to distribute, remix, adapt, build upon this work noncommercially, and license their derivative works on different terms, provided the original work is properly cited and the use is non-commercial. See: http:// creativecommons.org/licenses/by-nc/3.0/

\section{REFERENCES}

1. Cancer Research UK. Breast cancer incidence statistics [Internet]. Cancer Research UK; 2013 [cited 24 Jun 2013]. http://www. cancerresearchuk.org/cancer-info/cancerstats/types/breast/ incidence/uk-breast-cancer-incidence-statistics

2. NHS Information Centre. National Mastectomy and Breast Reconstruction Audit, Fourth Annual Report-2011-DatasetsDGU. 2011.

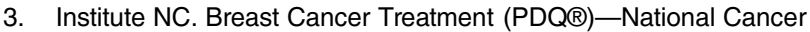
Institute [Internet]. 2013 [cited 6 Sep 2013]. http://www.cancer.gov/ cancertopics/pdq/treatment/breast/healthprofessional

4. Veronesi U, Banfi A, Saccozzi R, et al. Conservative treatment of breast cancer. A trial in progress at the Cancer Institute of Milan. Cancer 1977;39(6 Suppl):2822-6.

5. Veronesi U, Cascinelli N, Mariani L, et al. Twenty-year follow-up of a randomized study comparing breast-conserving surgery with radical mastectomy for early breast cancer. $N$ Engl $J$ Med 2002;347:1227-32

6. Veronesi U, Salvadori B, Luini A, et al. Breast conservation is a safe method in patients with small cancer of the breast. Long-term results of three randomised trials on 1,973 patients. Eur $J$ Cancer 1995;31A:1574-9.

7. LENT SOMA scales for all anatomic sites. Int $J$ Radiat Oncol Biol Phys 1995;31:1049-91.

8. Mioton LM, Smetona JT, Hanwright PJ, et al. Comparing thirty-day outcomes in prosthetic and autologous breast reconstruction: a multivariate analysis of 13,082 patients? J Plast Reconstr Aesthet Surg 2013;66:917-25.

9. Lymperopoulos NS, Sofos S, Constantinides J, et al. Blood loss and transfusion rates in DIEP flap breast reconstruction. Introducing a new predictor. J Plast Reconstr Aesthet Surg 2013. http://www.ncbi. $\mathrm{nlm}$.nih.gov/pubmed/?term=Blood+loss+and+transfusion+rates+in +DIEP+flap+breast+reconstruction.+Introducing+a+new+predictor.

10. Choices N. Breast implants-complications-NHS choices. Department of Health.

11. Avelar R. Allergan experience post approval studies. 2005. http:// www.fda.gov/downloads/AdvisoryCommittees/ CommitteesMeetingMaterials/MedicalDevices/ MedicalDevicesAdvisoryCommittee/ GeneralandPlasticSurgeryDevicesPanel/UCM270309.pdf
12. Reaby LL. Reasons why women who have mastectomy decide to have or not to have breast reconstruction. Plast Reconstr Surg 1998;101:1810-18.

13. Department of Health. Poly Implant Prothèse (PIP) silicone breast implants: review of the actions of the Medicines and Healthcare products Regulatory Agency (MHRA) and Department of Health. 2012.

14. Tanne JH. FDA warns about metal-on-metal hip replacements. BMJ 2013;346:f429.

15. Coleman SR. Structural fat grafting: more than a permanent filler. Plast Reconstr Surg 2006;118(3 Suppl):108S-20S.

16. Rigotti G, Marchi A, Galiè M, et al. Clinical treatment of radiotherapy tissue damage by lipoaspirate transplant: a healing process mediated by adipose-derived adult stem cells. Plast Reconstr Surg 2007:119:1409-22; discussion 1423-4.

17. Ersek RA, Chang P, Salisbury MA. Lipo layering of autologous fat: an improved technique with promising results. Plast Reconstr Surg 1998:101:820-6.

18. Hinderer UT, del Rio JL. Erich Lexer's mammaplasty. Aesthetic Plast Surg 1992;16:101-7.

19. Peer L. Loss of weight and volume in human fat grafts: with postulation of a "cell survival theory." Plast Reconstr Surg 1950;5:217-30.

20. Illouz YG. Body contouring by lipolysis: a 5-year experience with over 3000 cases. Plast Reconstr Surg 1983;72:591-7.

21. Bircoll M. Cosmetic breast augmentation utilizing autologous fat and liposuction techniques. Plast Reconstr Surg 1987;79:267-71.

22. Maillard GF. Liponecrotic cysts after augmentation mammaplasty with fat injections. Aesthetic Plast Surg 1994;18:405-6.

23. Gutowski KA. Current applications and safety of autologous fat grafts: a report of the ASPS fat graft task force. Plast Reconstr Surg 2009; $124: 272-80$

24. Khouri R, Del Vecchio D. Breast reconstruction and augmentation using pre-expansion and autologous fat transplantation. Clin Plast Surg 2009;36:269-80, viii.

25. ASPRS Ad-Hoc Committee on New Procedures. Report on autologous fat transplantation. Plast Surg Nurs 1987;7:140-1.

26. Coleman SR. Long-term survival of fat transplants: controlled demonstrations. Aesthetic Plast Surg 1995;19:421-5.

27. Coleman S. Structural fat grafting. St. Louis: Quality Medical Publishing, 2004.

28. Nguyen A, Pasyk KA, Bouvier TN, et al. Comparative study of survival of autologous adipose tissue taken and transplanted by different techniques. Plast Reconstr Surg 1990;85:378-86; discussion 387-9.

29. Illouz YG, Sterodimas A. Autologous fat transplantation to the breast: a personal technique with 25 years of experience. Aesthetic Plast Surg 2009;33:706-15.

30. Delay E, Garson S, Tousson G, et al. Fat injection to the breast: technique, results, and indications based on 880 procedures over 10 years. Aesthet Surg J 2009;29:360-76.

31. Spear SL, Wilson HB, Lockwood MD. Fat injection to correct contour deformities in the reconstructed breast. Plast Reconstr Surg 2005:116:1300-5.

32. Gimble JM, Floyd ZE. Fat circadian biology. J Appl Physiol 2009;107:1629-37.

33. Lin L, Fu X, Zhang X, et al. Rat adipose-derived stromal cells expressing BMP4 induce ectopic bone formation in vitro and in vivo. Acta Pharmacol Sin 2006;27:1608-15.

34. Yoshimura K, Sato K, Aoi N, et al. Cell-assisted lipotransfer for cosmetic breast augmentation: supportive use of adipose-derived stem/stromal cells. Aesthetic Plast Surg 2008;32:48-55; discussion 56-7.

35. Moseley TA, Zhu M, Hedrick MH. Adipose-derived stem and progenitor cells as fillers in plastic and reconstructive surgery. Plast Reconstr Surg 2006;118(3 Suppl):121S-8S.

36. Lu F, Li J, Gao J, et al. Improvement of the survival of human autologous fat transplantation by using VEGF-transfected adipose-derived stem cells. Plast Reconstr Surg 2009;124:1437-46.

37. Houghton J, Stoicov C, Nomura S, et al. Gastric cancer originating from bone marrow-derived cells. Science 2004;306:1568-71.

38. Wang WZ, Fang X-H, Williams SJ, et al. Analysis for apoptosis and necrosis on adipocytes, stromal vascular fraction, and adipose-derived stem cells in human lipoaspirates after liposuction. Plast Reconstr Surg 2013;131:77e-85e.

39. Pearl RA, Leedham SJ, Pacifico MD. The safety of autologous fat transfer in breast cancer: lessons from stem cell biology. J Plast Reconstr Aesthet Surg 2012;65:283-8.

40. Direkze NC, Jeffery R, Hodivala-Dilke K, et al. Bone marrow-derived stromal cells express lineage-related messenger RNA species. Cancer Res 2006;66:1265-9.

41. Wang $Y-Y$, Lehuédé $C$, Laurent $V$, et al. Adipose tissue and breast epithelial cells: a dangerous dynamic duo in breast cancer. Cancer Lett 2012;324:142-51. 
42. Bertolini F. Contribution of endothelial precursors of adipose tissue to breast cancer: progression-link with fat graft for reconstructive surgery. Ann Endocrinol (Paris) 2013;74:106-7.

43. Wang C-F, Zhou Z, Yan Y-J, et al. Clinical analyses of clustered microcalcifications after autologous fat injection for breast augmentation. Plast Reconstr Surg 2011;127:1669-73.

44. Shea BJ, Hamel C, Wells GA, et al. AMSTAR is a reliable and valid measurement tool to assess the methodological quality of systematic reviews. J Clin Epidemiol 2009;62:1013-20.

45. Shea BJ, Grimshaw JM, Wells GA, et al. Development of AMSTAR: a measurement tool to assess the methodological quality of systematic reviews. BMC Med Res Methodol 2007;7:10.

46. Shea BJ, Bouter LM, Peterson J, et al. External validation of a measurement tool to assess systematic reviews (AMSTAR). PLoS ONE 2007;2:e1350.

47. Leopardi D, Thavaneswaran P, Mutimer KLA, et al. Autologous fat transfer for breast augmentation: a systematic review. ANZ J Surg 2013. http://www.ncbi.nlm.nih.gov/pubmed/23656507

48. Claro FJ, Figueiredo JCA, Zampar AG, et al. Applicability and safety of autologous fat for reconstruction of the breast. Br J Surg 2012;99:768-80.

49. Rosing JH, Wong G, Wong MS, et al. Autologous fat grafting for primary breast augmentation: a systematic review. Aesthetic Plast Surg 2011;35:882-90.

50. Higgins JPT, Green S. eds. Cochrane Handbook for Systematic Reviews of Interventions Version 5.1.0 [updated March 2011] [Internet]. The Cochrane Collaboration. 2011 (cited 20 Jun 2013). http://www.cochrane-handbook.org

51. Moher D, Liberati A, Tetzlaff J, et al. Preferred reporting items for systematic reviews and meta-analyses: the PRISMA statement. Int $J$ Surg 2010;8:336-41.
52. Agha R, Goodacre T, Orgill D. Autologous fat grafting for breast reconstruction: a systematic review [Internet]. 2013 [cited 1 Aug 2013]. http://www.crd.york.ac.uk/PROSPERO/display_record.asp? ID=CRD42013005254\#.UhqAQIN8xa8

53. OCEBM Levels of Evidence Working Group. The Oxford 2011 levels of evidence [Internet]. Oxford: Centre for Evidence-

Based Medicine, 2011:5653. http://www.cebm.net/index.aspx?o=5653

54. Clavien PA, Barkun J, De Oliveira ML, et al. The Clavien-Dindo classification of surgical complications: five-year experience. Ann Surg 2009;250:187-96.

55. Netscher DT, Sharma S, Thornby J, et al. Aesthetic outcome of breast implant removal in 85 consecutive patients. Plast Reconstr Surg 1997;100:206-19.

56. Cano SJ, Klassen AF, Scott AM, et al. A closer look at the BREAST-Q(C). Clin Plast Surg 2013;40:287-96.

57. Google. Inside Google Translate-Google Translate [Internet]. http:// translate.google.com/about/intl/en ALL

58. Balk EM, Chung M, Chen ML, et al. Assessing the accuracy of Google Translate to allow data extraction from trials published in non-English languages 2013-PubMed-NCBI. 2013.

59. Coleman SR. In: Thorne CH. ed. Grabb \& Smith's plastic surgery. 6th edn. Philadelphia: Wolters Kluwer, 2007:480-5

60. Balshem $\mathrm{H}$, Helfand $\mathrm{M}$, Schünemann HJ, et al. GRADE guidelines: 3 . Rating the quality of evidence. J Clin Epidemiol 2011;64:401-6.

61. Higgins JPT, Green S. ed. 12.2.1 The GRADE approach [Internet]. Cochrane Handbook for Systematic Reviews of Interventions Version 5.1.0. 2011 [cited 24 Jun 2013]. http://handbook.cochrane. org/chapter_12/12_2_1_the_grade_approach.htm

62. Copenhagen: The Nordic Cochrane Centre, The Cochrane Collaboration. Review Manager (RevMan) Version 5.2. 2012. 